\title{
Manejo das Hemorragias Relacionadas aos Anticoagulantes Orais de Ação Direta
}

\author{
Management of Hemorrhage Related to Direct Action Oral Anticoagulant Medication
}

Isabela Ramos Ali Ganem 1, ${ }^{\text {, }}$ Luiz Claudio Behrmann Martins ${ }^{1}$, Carlos Eduardo Mendonça Tomé1,2

ORCID IDS

Ganem IRA (D) https://orcid.org/0000-0001-6340-991X

Martins LCB (D) https://orcid.org/0000-0003-4969-857X

Tomé CEM (D) https://orcid.org/0000-0001-7260-7483

\begin{abstract}
RESUMO
Introdução: Os anticoagulantes orais diretos (direct oral anticoagulant - DOACs) são uma nova classe de anticoagulantes que inibem diretamente a trombina (dabigatrana) ou o fator Xa (rivaroxabana, edoxabana e apixabana) na cascata da coagulação. Esses estão sendo cada vez mais utilizados para tratamento e prevenção de eventos tromboembólicos, principalmente em pacientes com fibrilação atrial, em substituição à varfarina ou outros antagonistas de vitamina K(AVKs). Embora a incidência de hemorragias seja maior nos AVKs do que nos DOACs, elas também podem ocorrer nesse grupo, até mesmo na forma de hemorragia intracraniana (HIC) com risco de morte. Atualmente as indicações dos DOACs vêm aumentando progressivamente, e a disponibilização de seus agentes reversores específicos certamente aumentará a segurança e, consequentemente, sua utilização. O idarucizumab, reversor da dabigatrana, e o andexanet alfa, reversor dos inibidores do fator Xa, foram aprovados pelo Food and Drug Administration (FDA) dos Estados Unidos e o ciraparantag poderá ser aprovado em um futuro próximo. Objetivo: Revisar a literatura sobre o manejo da hemorragia relacionada ao uso dos DOACs. Métodos: Revisão da literatura que utilizou artigos de 1998 a 2017, de diversas plataformas e revistas. Conclusão: Os DOACs constituem um grande avanço na profilaxia e tratamento da doença tromboembólica, que cursa com elevada morbimortalidade, e as hemorragias são os principais eventos adversos relacionados ao seu uso, sendo raramente necessária a reversão imediata da anticoagulação. No entanto, a existência dos reversores específicos dos DOAcs aumenta a segurança dos pacientes, que poderão ter sua anticoagulação revertida rapidamente, se necessário.
\end{abstract}

PALAVRAS-CHAVE: Fibrilação atrial; Coagulação sanguínea; Efeitos dos fármacos

\begin{abstract}
Introduction: Direct Oral Anticoagulant-DOACS) are a new class of anticoagulant that directly inhibit the trombine (dabigatran) or Xa factor (rivaroxabane, edoxabane and apixabane) in the coagulation cascade. These medications are being more frequently used for the treatment and prevention of thrombolytic events, mainly in patients with atrial fibrillation, in substitute to varfrine or other vitamin Kantagonists (VKAs). Although the incidence of hemorrhage is higher in AVKs than in DOACs, these events may also occur in this group, even in the form of intracranial hemorrhage (ICH), with risk of death. Nowadays, DOACs indications have progressively enhanced and the availability of their specific reverse agents certainly will enhance the safety of their usage. Idarucizumab, reverse agent of dabigatrane, and alpha andexanet, reverse agent of Xa factor, have been approved by the Food and Drug Administration in the United States and ciraparantag may be approved in a near future. Objective: To review the literature on the manage of hemorrhage related to the use of DOACs. Methods: Review of literature that used articles from 1998 to 2017, from several platforms and journals. Conclusion: DOACs constitute a great advance in prophylaxis and treatment of thrombolytic diseases, which presents elevated morbidymortality, and hemorrhages are the main adverse events related to their usage, being rarely necessary the immediate reverse of the anticoagulation. However, the existence of DOACs specific reverse agents enhance the safety of patients, whose anticoagulation may be rapidly reversed if necessary.
\end{abstract}

KEYWORDS: Atrial fibrillation; Blood coagulation; Pharmaceuticals effects.

1. Hospital Guilherme Alvaro - Santos/SP - Brasil.

2.Centro Universitário Lusiada - Santos/SP - Brasil.

*Autor correspondente: bela.ganem@hotmail.com

Recebido: 09 Jun 2019 | Aceito: 01 Jul 2019

Editor Associado: José Cláudio Kruse 


\section{INTRODUÇÃO}

A anticoagulação oral é fundamental para prevenção e tratamento de embolias e tromboses. Apenas nos Estados Unidos, mais de seis milhões de pessoas fazem uso de anticoagulantes orais (ACOs), o que pode se associar a maior risco de sangramento e repercutir na morbimortalidade desse grupo ${ }^{1}$.

A incidência das doenças que requerem anticoagulação oral vem aumentando progressivamente e isso está diretamente relacionado ao envelhecimento populacional. A fibrilação atrial (FA), a arritmia sustentada mais prevalente em todo o mundo, apresentou aumento de $13 \%$ em sua incidência nas últimas duas décadas, e a idade é fator de risco tanto para o desenvolvimento da FA quanto para aumento do risco de eventos cardioembólicos. $\mathrm{O}$ acidente vascular encefálico (AVE) é a principal complicação da FA e está relacionado a essa arritmia em $20 \%$ dos casos ${ }^{2}$.

Os ACOs de ação direta (direct oral anticoagulantDOACs), representados pelos inibidores diretos do fator $\mathrm{Xa}$ (rivaroxabana, edoxabana e apixabana) e o inibidor direto da trombina (dabigatrana), são alternativas aos antagonistas da vitamina $\mathrm{K}$ (AVKs) para profilaxia e tratamento de eventos tromboembólicos, em grande parte relacionados à FA e apresentam uma série de vantagens clínicas em comparação aos AVKs, como a maior estabilidade anticoagulante e menor risco tanto de hemorragia intracraniana quanto de sangramento ${ }^{3}$.

Com relação às complicações hemorrágicas, devido à curta meia-vida desses fármacos, na maioria dos casos apenas a sua suspensão, associada a medidas clínicas de suporte, pode ser suficiente para cessar o sangramento. A utilização de complexo protrombínico ${ }^{4}$, hemodiálise e carvão ativado (CA) podem ser úteis na redução da ação dos DOACs; no entanto, em casos de superdosagem, sangramentos maciços, comprometimento hemodinâmico ou necessidade de intervenção cirúrgica de urgência, pode ser necessária a reversão da atividade anticoagulante. Para atuar nesses casos mais graves foram desenvolvidos os reversores específicos dos DOACs, e as três principais drogas são: idarucizumabe, andexanet alfa e ciraparantag.

\section{OBJETIVO}

Revisar a literatura sobre o manejo das hemorragias relacionadas aos DOACs.

\section{MÉTODOS}

Este estudo de revisão de literatura utilizou artigos científicos publicados entre 1998 e 2017 pesquisados nas plataformas PubMed,Medline, Biblioteca Cochrane, SciELO e UpToDate e nas revistas científicas Journal of the American Medical Association, New England Journal of Medicine e Blood, utilizando as seguintes palavras-chave: fibrilação atrial, anticoagulação, DOAC, andexanet alfa, idarucizumabe, ciraparantag, atrial fibrillation e anticoagulation.

\section{DESENVOLVIMENTO}

A hemostasia é uma resposta fisiológica normal do organismo que previne perdas sanguíneas significativas após um dano vascular. A cascata da coagulação é uma série complexa de reações que garantem a ocorrência da hemostasia (Fig. 1).

O sistema anticoagulante, que trabalha em paralelo com o sistema fibrinolítico, garante que a formação de trombo seja um processo controlado e equilibrado, permitindo a degradação de trombos já formados. Os DOACs são divididos em 2 grupos, de acordo com o local de ação na cascata da coagulação, podendo ser inibidores diretos da trombina ou inibidores diretos do fator $\mathrm{Xa}$, e suas características são apresentadas na Tabela 1

A dabigatrana exetilato é um pró-fármaco de administração oral que é convertido no fígado em dabigatran, um inibidor direito, reversível e competitivo da trombina ${ }^{5}$. Existem testes de atividade anticoagulante, como o teste de coagulação de ecarina (ecarin clotting time - ECT), tempo de trombina (TT) e tempo de tromboplastina parcial ativada (TTPa) para detectar a atividade excessiva da dabigatrana. O TT é o mais sensível para detectar baixos níveis de dabigatrana ${ }^{6,7}$.

Os inibidores diretos do fator $\mathrm{Xa}$ (rivaroxabana, apixabana e edoxabana) ligam-se diretamente aos sítios ativos do fator $\mathrm{X}$ na circulação e, ligados ao coágulo, bloqueiam a interação com o seu substrato. São metabolizados pelos rins (25-35\%) e fígado (até 75\%) e, pacientes com insuficiência hepática, podem apresentar acúmulo da droga no organismo, mas, apesar disso, não são hepatotóxicos ${ }^{8}$.

A rivaroxabana é um inibidor direto, reversível e competitivo do fator $\mathrm{Xa}$ da coagulação, de administração via oral. Não deve ser usada em pacientes com clearence $<15 \mathrm{~mL} / \mathrm{min}$, assim como em pacientes com doença hepática importante (Child-Pugh B e C com coagulopatia) ${ }^{9}$. A medicação não foi testada em jovens com menos de 18 anos $^{10}$. 


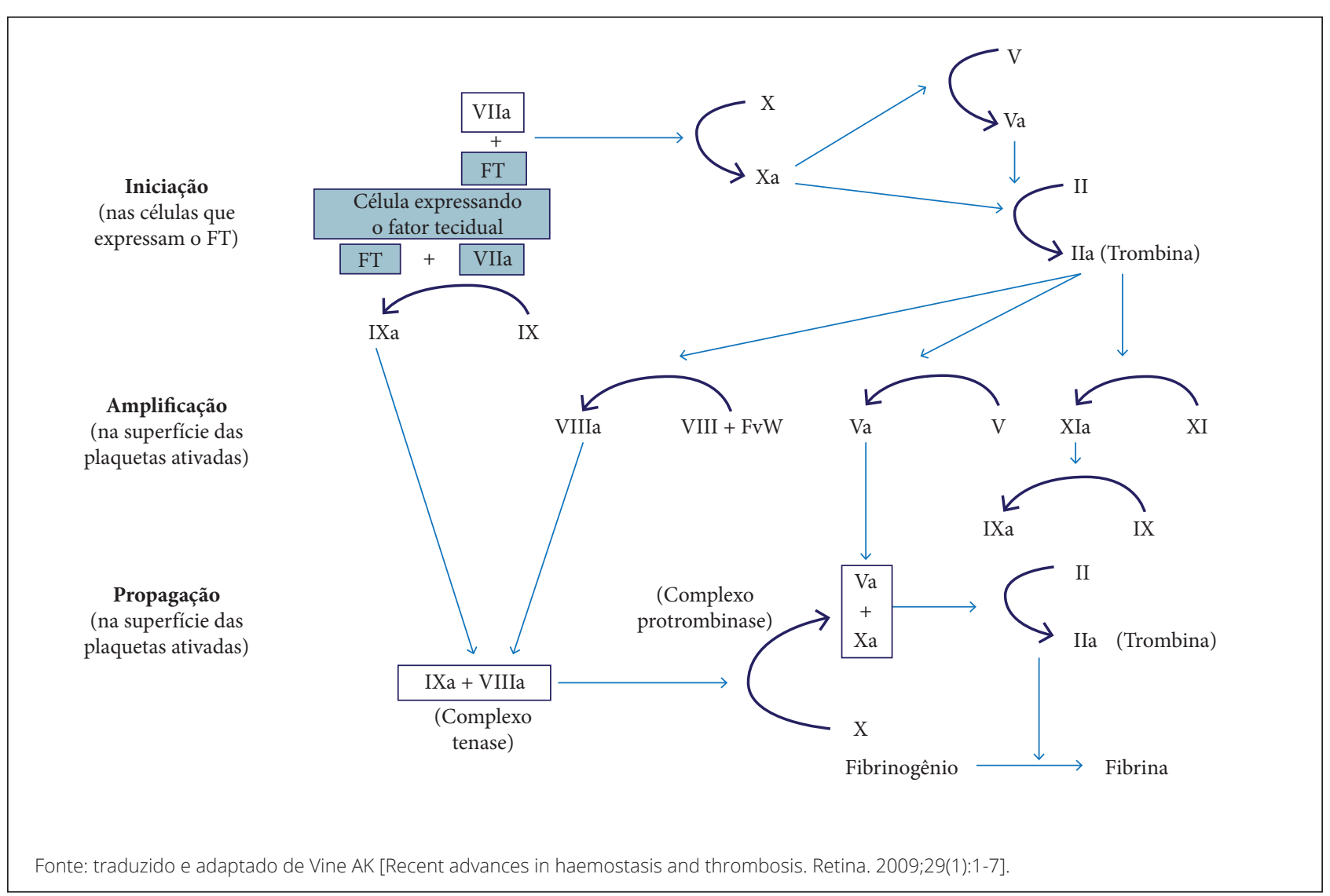

Figura 1. Cascata da coagulação [fator tecidual (FT) ativado].

A rivaroxabana interage com as drogas inibidoras do CYP-3A4 e da proteína P (p. ex., cetoconazol, itraconazol, voriconazol, posaconazol e ritonavir) e seu uso concomitante com essas drogas é contraindicado ${ }^{11}$. Indutores potentes do CYP-3A4 (p. ex., rifampicina e carbamazepina) podem reduzir o efeito da medicação ${ }^{12}$. Não é necessário realizar exames para monitorar a coagulação durante o tratamento com rivaroxabana, exceto em pacientes que apresentem sangramento, suspeita de intoxicação pelo medicamento ou necessidade de cirurgia de emergência ${ }^{13}$.

A apixabana é um inibidor direto, reversível e competitivo do fator Xa, administrado por via oral, necessitando de ajuste de sua dose de acordo com o clearence de creatinina. Também se recomenda reduzir sua dose em pacientes que fazem uso de inibidores da CYP-3A4 e proteína $\mathrm{P}^{14}$.

A edoxabana é um inibidor direto do fator $\mathrm{Xa}$, seletivo e reversível ${ }^{15,16}$. Em torno de $73 \%$ da dose da droga é excretada inalterada nas fezes e na urina ${ }^{17,18}$. A dose recomendada para prevenção de acidente vascular encefálico em pacientes com FA é de $60 \mathrm{mg}$ uma vez ao dia, reduzindo para $30 \mathrm{mg}$ uma vez ao dia em pacientes com clearence de creatinina entre $15-50 \mathrm{~mL} / \mathrm{min}$, peso $<60 \mathrm{~kg}$ ou uso concomitante de algum dos inibidores da proteína $\mathrm{P}$ (p. ex., verapamil, quinidina, eritromicina e cetoconazol $)^{19}$. A medicação não é recomendada para pacientes com doença renal terminal, clearence de creatinina $<15 \mathrm{~mL} / \mathrm{min}$ ou realizando hemodiálise ${ }^{20}$.

$A$ incidência de sangramento maior em pacientes utilizando DOACs é de 2-3\% ao ano, sendo a incidência de AVE hemorrágico de $0,1-0,5 \%{ }^{21}$.

\section{DISCUSSÃO}

O manejo das hemorragias relacionadas ao uso dos DOACs varia conforme a gravidade do caso. Hemorragias leves podem ser resolvidas apenas com a descontinuação temporária da droga, e em casos mais graves, podem ser necessários à reposição de fluidos, o suporte hemodinâmico, a compressão mecânica, a hemostasia cirúrgica e a utilização de produtos derivados do sangue. Se essas medidas não forem suficientes, deve-se utilizar pró-coagulantes, como o complexo protrombínico ${ }^{21}$.

Outras terapias incluem a hemodiálise, capaz de remover até $60 \%$ da dabigatrana circulante, e o CA, eficaz em reduzir 
Tabela 1. Propriedades dos anticoagulantes orais (ACOs) diretos.

\begin{tabular}{|c|c|c|c|c|}
\hline & Dabigatrana & Rivaroxabana & Apixabana & Endoxabana \\
\hline $\begin{array}{l}\text { Mecanismo } \\
\text { de ação }\end{array}$ & $\begin{array}{l}\text { Inibidor direto da } \\
\text { trombina }\end{array}$ & Inibidor do fator Xa & Inibidor do fator $\mathrm{Xa}$ & Inibidor do fator Xa \\
\hline $\begin{array}{c}\text { Tempo para atingir } \\
\text { nível sérico }\end{array}$ & $\begin{array}{l}1 \text { a } 2 \text { h se ingerido } \\
\text { com alimentação }\end{array}$ & $2 \mathrm{a} 4 \mathrm{~h}$ & $3 a 4 h$ & $1 \mathrm{a} 2 \mathrm{~h}$ \\
\hline Meia-vida & $\begin{array}{l}12 \text { a } 17 \text { h (jovens); } \\
14 \text { a } 17 \text { h (idosos) }\end{array}$ & $\begin{array}{l}5 \text { a } 9 \text { h (jovens); } \\
11 \text { a } 13 \text { h (idosos) }\end{array}$ & $12 \mathrm{~h}$ & $10 \mathrm{a} 14 \mathrm{~h}$ \\
\hline Dose (fanv) & 150 mg 2 vezes ao dia & 20 mg 1 vez ao dia & 5 mg 2 vezes ao dia & 60 mg 1 vez ao dia \\
\hline $\begin{array}{c}\text { Metabolismo } \\
\text { pelo cyp3a4 }\end{array}$ & Não & Sim & Sim & Mínimo \\
\hline Eliminação & $80 \%$ renal & $\begin{array}{l}\text { 70\% hepática } \\
\text { 30\% renal }\end{array}$ & $30 \%$ renal & $50 \%$ renal \\
\hline
\end{tabular}

Fonte: Crit Care Res Pract. 2018. ID:4907164.

a absorção dos anticoagulantes nas primeiras horas após a ingestão. Nos casos de superdosagem, sangramentos maciços, necessidade de restauração da hemostasia por comprometimento hemodinâmico ou necessidade de intervenção cirúrgica de urgência, pode ser necessária a reversão da atividade anticoagulante utilizando os reversores dos $\mathrm{DOACs}^{21}$. Suas indicações e características são apresentadas nas Tabelas 2 e 3.

O idarucizumabe, reversor específico da dabigatrana, foi aprovado para uso clínico em outubro e novembro de 2015, nos Estados Unidos e na Europa, respectivamente, e no Brasil a liberação ocorreu em abril de 2017. O andexanet alfa, reversor dos inibidores do fator $\mathrm{Xa}$, foi aprovado pelo Food and Drug Administration (FDA) em esquema acelerado, porém no Brasil ainda não foi liberado para uso. O mais recente reversor dos DOACs, o ciraparantag, está em fase inicial de estudos e propõe a reversão de todos os tipos de DOACs.

\section{Medidas clínicas}

Em caso de sangramento maior, que ocorre em local crítico, acompanhado de instabilidade hemodinâmica ou queda $\pm 2 \mathrm{~g} / \mathrm{dL}$ de hemoglobina, os DOACs devem ser suspensos, sendo indicados os seus reversores específicos associados à reposição volêmica e hemostasia local ${ }^{22}$.

Para sangramentos exteriorizados devem ser feitas medidas locais de controle em associação com a expansão volêmica agressiva, preferencialmente com cristaloides (soro fisiológico a 0,9\% ou Ringer com Lactato), visando à restauração volêmica e à estabilidade hemodinâmica. Tanto a hipotermia quanto a acidose devem ser corrigidas e, em casos de anemia sintomática e/ou sangramento ativo, devem ser transfundidas hemácias, visando manter a hemoglobina $\pm 7 \mathrm{~g} / \mathrm{dL}$ nos pacientes gerais e $\pm 8 \mathrm{~g} / \mathrm{dL}$ nos pacientes coronarianos ${ }^{23}$. A transfusão de plaquetas deve ser feita se houver menos do que $50.000^{24}$ e de crioprecipitado se fibrinogênio for menor do que $100 \mathrm{mg} / \mathrm{dL}$. Deve-se ter cuidado ao indicar DOACs para pacientes com comorbidades que aumentem o risco de sangramento, principalmente a dabigatrana, pois a disfunção hepática pode levar à coagulopatia e à redução do metabolismo do anticoagulante, aumentando, assim, a chance de hemorragias. $\mathrm{Na}$ indisponibilidade dos reversores específicos dos DOACs, devem ser utilizadas medidas reversoras não específicas.

O complexo protrombínico é um complexo de fatores derivados do plasma humano capaz de reverter a anticoagulação dos AVKs. Em estudos em animais de experimentação, o complexo protrombínico também foi eficaz na reversão do efeito da dabigatrana ${ }^{25}$, com dose recomendada de $50 \mathrm{U} / \mathrm{kg}$ (máxima de $4.000 \mathrm{U})^{26}$. Não há estudos randomizados avaliando o uso de complexo protrombínico em pacientes com sangramento maior associado ao uso de inibidores do fator-Xa, portanto a dose é baseada em relatos de casos, séries de casos e estudos em fase pré-clínica, sendo a dose inicial sugerida de $50 \mathrm{U} / \mathrm{kg}^{27}$. O CA pode ser utilizado para remover a droga ainda não absorvida pelo trato gastrointestinal, devendo ser realizada em até 2 a 4 horas após a ingestão ${ }^{28}$. 
Tabela 2. Indicações do uso dos reversores dos anticoagulantes orais diretos (direct oral anticoagulant - DOACs).

Sangramento com risco de morte: hemorragia intracraniana, hemorragia extradural sintomática ou em expansão ou hemorragia incontrolável

Sangramento "fechado" ou em órgão críticos: intraespinhal, intraocular, pericárdico, pulmonar, retroperitoneal ou intramuscular com síndrome compartimental

Sangramento maior persistente apesar de medidas para hemostasia local ou risco de sangramento recorrente

Cirurgia ou intervenção de emergência em pacientes com alto risco de sangramento durante o procedimento: neurocirurgia, punção lombar, cirurgia cardíaca, vascular e hepática

Fonte: J Thromb Haemost. 2015;14:623-7.

Tabela 3. Propriedades dos reversores específicos dos anticoagulantes orais diretos.

\begin{tabular}{|c|c|c|c|}
\hline & Idarucizumabe & Andexanete alfa & Ciraparantag \\
\hline Alvo & Dabigratana & $\begin{array}{l}\text { Inibidor diretos do fator Xa, } \\
\text { HBPM, fondaparinux }\end{array}$ & $\begin{array}{l}\text { Inibidores diretos fator Xa, } \\
\text { heparina de baixo peso } \\
\text { molecular, fondaparinux, } \\
\text { heparina, dabigratana }\end{array}$ \\
\hline Composto & $\begin{array}{l}\text { Fragmento de anticorpo } \\
\text { monoclonal humanizado }\end{array}$ & $\begin{array}{l}\text { Molécula recombinte derivado } \\
\text { do fator Xa humano }\end{array}$ & $\begin{array}{l}\text { Ligação ao alvo por meio de } \\
\text { ligações não covalentes de } \\
\text { hidrogênio, diminuindo sua } \\
\text { ligação aos alvos endógenos }\end{array}$ \\
\hline Mecanismo de ação & $\begin{array}{c}\text { Afinidade de ligação com a } \\
\text { dabigatrana } 350 \text { vezes maior } \\
\text { do que a afinidade de ligação } \\
\text { dabigratana-trombina }\end{array}$ & $\begin{array}{l}\text { Alteração do receptor dos } \\
\text { inibidores do fator Xa com } \\
\text { maior afinidade de ligação que } \\
\text { o fator Xa "natural" }\end{array}$ & 100-400 mg intravenoso \\
\hline Dose & $\begin{array}{c}5 \mathrm{~g} \text { (divididos em duas doses de } \\
2,5 \mathrm{~g} \text { intravenosas) }\end{array}$ & $\begin{array}{c}\text { 400-800 mg em bolus, seguidos } \\
\text { por 4-8 mg/min em infusão } \\
\text { contínua em } 2 \mathrm{~h}\end{array}$ & Dentro de $10 \mathrm{~min}$ \\
\hline Início de ação & Imediato & Dentro de 5 min & $\operatorname{sim}$ \\
\hline Duração de reversão & $12 \mathrm{~h}$ & $1 \mathrm{a} 2 \mathrm{~h}$ & $24 \mathrm{~h}$ \\
\hline Eliminação & Renal & Indefinido & Indefinido \\
\hline Estudo clínico & Reverse-AD & Annexa-A e AnnexA-R & Ansell et $a^{37}$. \\
\hline Fase de desenvolvimento & III/aprovado & III & $\|$ \\
\hline Efeitos adversos & $\begin{array}{l}\text { Reação cutânea, hematomas no } \\
\text { sítio de aplicaçãoe epistaxe }\end{array}$ & $\begin{array}{l}\text { Urticária, disgeusia, cefaleia e } \\
\text { ruborização }\end{array}$ & $\begin{array}{l}\text { Disgeusia, cefaléia e } \\
\text { ruborização }\end{array}$ \\
\hline
\end{tabular}

HBPM: heparina de baixo peso molecular

Fonte: Critical Care Research and Practice. 2018. ID: 4907164.

A hemodiálise é útil em sangramentos relacionados à dabigatrana, devido à baixa afinidade dessa molécula pelas proteínas plasmáticas, sendo a excreção predominantemente renal ${ }^{29}$.Já os inibidores do fator Xa não podem ser dialisados, pois apresentam forte ligação com as proteínas plasmáticas ${ }^{21}$.
Agentes antifibrinolíticos podem ser indicados para pacientes com sangramento maior ocasionado tanto por inibidores do fator Xa quanto pela dabigatrana. Deve-se evitar o uso empírico de fator VII ativado, plasma fresco congelado ou crioprecipitado devido à falta de estudos 
demonstrando benefício e aos elevados riscos associados (p. ex., reação transfusional, trombose, sobrecarga de volume) ${ }^{21}$.

Os reversores dos DOACs estão indicados em casos de necessidade de reversão urgente do efeito anticoagulante, como em sangramentos maciços, na presença de instabilidade hemodinâmica ou quando o paciente necessita de cirurgia de urgência/emergência na vigência do uso desses fármacos.

$\mathrm{O}$ idarucizumabe pode ser utilizado para reverter o efeito da dabigatrana. Ele é derivado de um fragmento de anticorpo monoclonal humanizado produzido em células de ovário de hamsters da China e se liga à dabigatrana com alta afinidade e especificidade. $\mathrm{O}$ reconhecimento e a ligação com a dabigatrana se dão devido à similaridade estrutural com a trombina, mediada por interações hidrofóbicas, ligações de hidrogênio e pontes salinas. Não é necessário ajuste de dose de acordo com a função renal e hepática, peso ou idade. $\mathrm{O}$ pico de concentração de idarucizumabe é atingido em poucos minutos, seguido de rápida eliminação $0^{30}$.

Administrando-se $5 \mathrm{~g}$ de idarucizumabe, 32,1\% será recuperada na urina no período de 6 horas e menos de 1\% nas próximas 18 horas. O restante é eliminado por meio de catabolismo proteico, principalmente pelos rins. Observou-se proteinúria transitória, que normalmente atinge o pico 4 horas após sua administração e desaparece entre 12-24 horas. $\mathrm{Na}$ ausência da dabigatrana no organismo, o idarucizumabe não tem nenhum efeito sobre a trombina ou os parâmetros de coagulação (dTT,ECT,TT,TTPa).Em estudos de Fase 1 não foram demonstradas diferenças estatisticamente significantes entre os efeitos colaterais encontrados em pacientes que receberam placebo ou idarucizumabe, e também não foram reportados achados clínicos relevantes ou alterações de parâmetros laboratoriais, sinais vitais, eletrocardiograma ou exame físico ${ }^{3}$. A seleção da dose de idarucizumabe para uso em testes clínicos foi baseada em metas para neutralizar a dose de dabigatrana utilizada em pacientes com FA no estudo RE-LY (150 mg de dabigatrana duas vezes ao dia). A dose de $5 \mathrm{mg}$ foi escolhida para reverter a dose do anticoagulante em pacientes com disfunção renal moderada. Nos estudos que envolveram pacientes tanto do sexo masculino quanto do feminino de diferentes idades e níveis de função renal, a administração de idarucizumabe, na dose de 2,5 g de 12/12 horas, resultou na reversão satisfatória da anticoagulação pela dabigatrana, sendo essa a dose selecionada para estudos posteriores. O idarucizumabe mostrou-se eficaz e seguro na reversão do efeito anticoagulante da dabigatrana, em situações de sangramento. A segurança observada no estudo dá suporte para o seu uso em situações de emergência, e a medicação já se encontra nas recomendações para o tratamento de hemorragias graves ${ }^{31}$.

$\mathrm{O}$ andexanet alfa é um reversor específico dos inibidores do fator Xa criado por bioengenharia. É uma molécula recombinante com estrutura semelhante à do fator Xa endógeno, com alta afinidade pelos inibidores do fator $\mathrm{Xa}$, tanto diretos quanto indiretos (rivaroxabana, edoxabana, apixabana, heparina de baixo peso molecular e fondaparinux), mas não tem atividade catalítica, não realizando a clivagem de protrombina em trombina. $\mathrm{O}$ sítio de ligação serina do fator Xa foi substituído por alanina, o que permite a ligação e sequestro dos inibidores do fator Xa no intravascular, restaurando assim a atividade do fator $\mathrm{Xa}$ endógeno, com consequente redução da atividade anticoagulante ${ }^{32}$.

$\mathrm{O}$ andexanet alfa deve ser administrado por via intravenosa, sendo a primeira dose em bolus, seguida por uma dose de manutenção nas próximas 2 horas $^{33}$. O início da ação se dá em 2 a 5 minutos após a infusão da droga e sua meia-vida é de 30 a 60 minutos. Os níveis séricos dos anticoagulantes inibidores do fator Xa voltam a subir após algumas horas da administração de andexanet alfa, e a droga não é removida com o agente reversor, como ocorre com a dabigatran, ao se utilizar o anticorpo idarucizumabe ${ }^{34,35}$. Não é necessário ajuste da dose, e aproximadamente 2 minutos após a administração, o andexanet alfa demonstrou reversão dos efeitos anticoagulantes de todos os inibidores do fator $\mathrm{Xa}$, incluindo redução da sua atividade e restauração da geração de trombina e tempo de coagulação. A administração endovenosa em bolus, seguida da infusão contínua de andexanet, resultou numa redução sustentada da atividade do anti-fator $\mathrm{Xa}$, a qual retornou aos níveis do grupo placebo 2 horas após a parada da infusão $0^{34}$

O ciraparantag (PER977) é uma molécula sintética pequena e catiônica que se liga aos inibidores do fator $\mathrm{Xa}$, inibidores diretos da trombina, heparina não fracionada $\mathrm{e}$ heparina de baixo peso molecular por meio de ligações de hidrogênio não covalentes. Estudos Fase 2 investigando reversão de edoxabana e avaliando as doses do ciraparantag estão em andamento e planos para estudos Fase 3 já foram anunciados ${ }^{36}$.

\section{CONCLUSÃO}

Os DOACs constituem um grande avanço na profilaxia e tratamento da doença tromboembólica que cursa com 
elevada morbimortalidade. Essas drogas são de fácil utilização, apresentam grande eficácia e segurança e não necessitam de ajustes terapêuticos pelo coagulograma, o que vem levando a um aumento progressivo na sua utilização. Um obstáculo importante à utilização dos DOACs era a impossibilidade de reversão do seu efeito anticoagulante em caso de sangramentos graves ou que necessitassem de cirurgias de emergência.

Apesar de raramente ser necessária essa reversão imediata, a existência dos reversores dos DOACs aumenta a segurança dos pacientes, podendo levar a aumento da sua utilização. Por esse motivo é tão importante a disponibilização dos reversores específicos dos DOACs mesmo sabendo que a maioria dos sangramentos relacionados ao seu uso não necessita de nenhuma intervenção drástica.

\section{CONTRIBUIÇÃO DOS AUTORES}

Conceituação, Ganem IRA; Martins LCB e Tomé CEM; Metodologia, Ganem IRA e Tomé CEM; Investigação, Ganem IRA; Martins LCB e Tomé CEM; Redação - primeira versão, Ganem IRA; Martins LCB e Tomé CEM; Redação - revisão \& edição, Ganem IRA; Martins LCB e Tomé CEM; Aquisição de financiamento, Ganem IRA; Martins LCB e Tomé CEM; Recursos, Ganem IRA; Martins LCB e Tomé CEM; Supervisão, Martins LCB e Tomé CEM..

\section{REFERÊNCIAS}

1. Barnes GD, Lucas E, Alexander GC, Goldberger ZD. National trends in ambulatory oral anticoagulant use. Am J Med. 2015;128(12):1300-5.e2. https://doi.org/10.1016/j. amjmed.2015.05.044

2. Mozaffarian D, Benjamin EJ, Go AS, Arnett DK, Blaha MJ, Cushman M, et al. Heart disease and stroke statistics-2016 update: a report from American Heart Association. Circulation. 2016;133:e38-360. https://doi.org/10.1161/ CIR.0000000000000350

3. Reilly PA, van Ryn J, Grottke O, Glund S, Stangier J. Idarucizumab, a specific reversal agent for Dabigatran: Mode of action, Pharmacokinetics and pharmacodynamics, and safety and efficacy in fase 1 subjects. The American Journal of Medicine. 2016. http://dx.doi.org/10.1016/J. amjmed.2016.06.007

4. Suryanarayan D, Schulman S. Potencial antidotos for reversal of old and new oral anticoagulants. Tirombaço Res. 2014;133(Suppl 2):S158-66. https://doi.org/10.1016/ S0049-3848(14)50026-6

5. Marques SP. Velhos e novos anticoagulantes orais. Perspectiva farmacológica. Rev Port Cardiol. 2012;31(1):616. https://doi.org/10.1016/S0870-2551(12)70034-3

6. Dager WE, Gosselin RC, Kitchen S, Dwyre D. Dabigatran effects on the international normalized ratio, activated partial thromboplastin time, thrombin time, and fibrinogen: a multicenter, in vitro study. Ann Pharmacother. 2012;46:1627. https://doi.org/10.1345/aph.1R179

7. Reilly PA, Lehr T, Haertter S, Conolly SJ, Yusuf S, Eikelboom JW, et al. The effect of dabigatran plasma concentrations and patient characteristics on the frequency of ischemic stroke and major bleeding in atrial fibrillation patients: the RE-LY Trial (Randomized Evaluation of Long-Term Anticoagulation Therapy). J Am Coll Cardiol. 2014;63:321-38. https://doi. org/10.1016/j.jacc.2013.07.104
8. Douros A, Azoulay L, Yin H, Suissa S, Renoux C. Non-vitamin K antagonist oral anticoagulants and risk of serious liver injury. J Am Coll Cardiol. 2018;71:1105. https://doi.org/10.1016/j. jacc.2018.01.009

9. Poulsen BK, Grove EL, Husted SE. New oral anticoagulants: a review of the literature with particular emphasis on patients with impaired renal function. Drugs. 2012;72:1739. https:// doi.org/10.2165/11635730-000000000-00000

10. Turpie AG, Kreutz R, Llau J, Norrving B, Haas S. Management consensus guidance for the use of rivaroxaban - an oral, direct factor Xa inhibitor. Thromb Haemost. 2012;108:876. https://doi.org/10.1160/TH12-03-0209

11. Gulseth MP, Wittkowsky AK, Fanikos J, Spinler SA, Dager WE, Nutescu EA, et al. Dabigatran etexilate in clinical practice: confronting challenges to improve safety and effectiveness. Pharmacotherapy. 2011;31:1232. https://doi.org/10.1592/ phco.31.12.1232

12. Nutescu E, Chuatrisorn I, Hellenbart E. Drug and dietary interactions of warfarin and novel oral anticoagulants: an update. J Thromb Thrombolysis. 2011;31:326. https://doi. org/10.1007/s11239-011-0561-1

13. Powell JR. Are new oral anticoagulant dosing recommendations optimal for all patients? JAMA. 2015;313:1013. https://doi.org/10.1001/jama.2015.59

14. Bristol-Myers Squibb Pharma Company. Highlights of prescribing information. Disponível em: http:// packageinserts.bms.com/pi/pi_eliquis.pdf

15. Furugohri T, Isobe K, Honda Y, Kamisato-Matsumoto C, Sugiyama N, Nagahara T, et al. DU-176b, a potent and orally active fator $\mathrm{Xa}$ inhibitor: in vitro and in vivo pharmacological profiles. Thromb Haemost. 2008;6(9):1542-9. https://doi. org/10.1111/j.1538-7836.2008.03064.x 
16. Zafar MU, Vorchheimer DA, Gaztanaga J, Velez M, Yadegar D, Moreno PR, et al. Antithrombotic effects of factor Xa inhibition with DU-176b: Phase-1 study of an oral, direct factor Xa inhibitor using an ex-vivo flow chamber. Thromb Haemost. 2007;98(4):883-8. https://doi.org/10.1160/TH0704-0312

17. Lixiana. Summary of product characteristics. (European Medicines Agency). Disponível em: http://www.ema. europa.eu/ema/index.jsp?curl=Pages/medicines/ human/medicines/002629/human_med_001874. jsp\&mid=WC0b01ac058001d124

18. Masumoto H, Yoshigae $Y$, Watanabe $K$, Takakusa H, Okazaki O, Izumi T, et al. In vitro metabolism of edoxaban and the enzymes involved in the oxidativo metabolism of edoxaban. AAPS J. 2010;12:1

19. Salazar DE, Mendell J, Kastrissios H, Green M, Carrothers $\mathrm{T}$, Song $\mathrm{S}$, et al. Modelling and simulation of edoxaban exposure and response relationships in patients with atrial fibrillation. Thromb Haemost 2012;107(5):925-36. https:// doi.org/10.1160/TH11-08-0566

20. Ruff CT, Giugliano RP, Antman EM, Crugnale SE, Bocanegra $T$, Mercuri $M$, et al. Evaluation of the novel factor Xa inhibitor edoxaban compares with warfarin in patients with atrial fibrillation: design and rationale for the Effective aNticoaGulation with factor XA next GEneration in Atrial Fibrillation - Thrombolysis In Myocardial Infarction study 48 (ENGAGE AF-TIMI 48). Am Heart J. 2010;160(4):635-41. https://doi.org/10.1016/j.ahj.2010.06.042

21. Hylek EM, Held C, Alexander JH, et al. Major bleeding in patients with atrial fibrillation receiving apixaban or warfarin: The ARISTOTLE Trial (Apixaban for Reduction in Stroke and Other Thromboembolic Events in Atrial Fibrillation): Predictors, Characteristics, and Clinical Outcomes. J Am Coll Cardiol. 2014;63:2141. https://doi.org/10.1016/j. jacc.2014.02.549

22. Garcia DA, Crowther M. Management of bleeding in patients receiving direct oral anticoagulants. UpToDate.2018

23. Carson JL, Guyatt G, Heddle NM, Grossman BJ, Cohn CS, Fung MK, et al. Clinical practice guidelines from the AABB: red blood cell transfusion thresholds and storage. JAMA. 2016;316:2025-35. https://doi.org/10.1001/jama.2016.9185

24. Contreras M. Consensus conferenc on platelet transfusion. Final statement. Blood Rev. 1998;12:239-40. https://doi. org/10.1016/S0268-960X(98)90005-4

25. Tummala R, Kavtaradze A, Gupta A, Ghosh RK.Specific antidotes against direct oral anticoagulants-a comprehensive review of clinical trials data. Int J Cardiol. 2016;214:292-8. https://doi.org/10.1016/j.ijcard.2016.03.056

26. Wong $H$, Keeling D. Activate prothrombin complex concentrate for the prevention of dabigatran-associated bleeding. $\mathrm{Br} J$ Haematol. 2014;166:152-3. https://doi. org/10.1111/bjh.12831

27. Dibu JR, Weimer JM, Ahrens C, Manno E, Frontera JA.The role of FEIBA in reversing novel oral anticoagulants in intracerebral hemorrhage. Neurocrit Care. 2016;24:413-9. https://doi.org/10.1007/s12028-015-0213-y

28. Van Ryn J, Stangier J, Haertter S, Liesenfeld KH, Wienen W, Feuring M. Dabigatran etexilate-a novel, reversible, oral direct thrombin inhibitor: interpretation of coagulation assays and reversal of anticoagulant activity. Thromb Haemost. 2010;103:1116-27. https://doi.org/10.1160/ TH09-11-0758

29. Stangier J, Rathgen $\mathrm{K}$, Stahle $\mathrm{H}_{\text {, et al. Influence of renal }}$ impairmentonthepharmacokineticsand pharmacodynamics of oral dabigatran etexilate. Clin Pharmaokinet. 2010;49:25968. https://doi.org/10.2165/11318170-000000000-00000

30. Schiele F, van Ryn J, Canada K, Newsome C, Sepulveda E, Park J, Nar $\mathrm{H}$, et al. A specific antidote for dabigatran, funcional and structural characterization. Blood. 2013;121:3554-62. https://doi.org/10.1182/blood-2012-11-468207

31. Glund S, Stangier J, Schmohl M, Ganser D, Norris S, van Ryn J, et al. Safety, tolerability, and efficacy of idarucizumab for the reversal of the anticoagulant effect of dabigatran in healthy male volunteers: a randomised, placebo-controlled, doubleblind phase 1 trial. Lancet. 2015;386:680-90. https://doi. org/10.1016/S0140-6736(15)60732-2

32. Lu G, DeGuzman FR, Hollenbach SJ, Karbarz MJ, Abe K, Lee $G$, et al. A specific antidote for reversal of anticoagulation by direct and indirect inhibitors of coagulation factor Xa. Nat Med. 2013;19:446-51. https://doi.org/10.1038/nm.3102

33. Connolly SJ, Milling TJ, Eikelboom JW, Gibson CM, Gold A, Curnutte JT, et al. Andexanet alfa for acute major bleeding associated with factor Xa inhibitors. N Engl J Med. 2016;375(12):1131-41. https://doi.org/10.1056/ NEJMoa1607887

34. Siegal DM, Curnutte JT, Connolly SJ, Lu G, Conley PB, Wiens $\mathrm{BL}$, et al. Andexanet alfa for the reversal of factor Xa inhibitor activity. N Engl J Med. 2015;373(25):2413-24. https://doi. org/10.1056/NEJMoa1510991

35. Quintard H, Viard D, Drici MD, Ruetsch C, Samama CM, Ichai C. Idarucizumab administration for reversing dabigatran effect in na acute kidney injured patient with bleeding. Thromb Haemost. 2017;117(1):196-7. https://doi.org/ 10.1160/TH16-06-0445

36. Ansell JE, Bakhru SH, Laulicht BE, Steiner SS, Grosso M, Brown K, Dishy V, et al. Use of PER977 to reverse the anticoagulants effect of edoxaban. N Engl J Med 2014; 371: 2141-2

37. J. E. Ansell, S. H. Bakhru, B. E. Laulicht et al., "Use of PER977 to reverse the anticoagulant effect of edoxaban," New England Journal of Medicine, vol. 371, no. 22, pp. 2141-2142, 2014 